\title{
Faculty views of and expectations for dimensional analysis
}

\author{
MacKenzie Lenz, and Elizabeth Gire \\ Department of Physics, Oregon State University, Corvallis, OR 97331
}

\begin{abstract}
Although dimensional analysis is a fundamental skill in physics, very few studies examine how instructors teach it or how they expect their students to do it. In this phenomenographic study, we interviewed eight faculty from Oregon State University with diverse teaching and research backgrounds to better understand how they think about using and teaching dimensional analysis. The faculty identified the term "dimensional analysis" as referring to two different processes - using dimensions to derive equations or using units/dimensions to check for errors. The latter process is also referred to as "unit checking". The faculty expect students at all levels to be able to check units/ dimensions. The faculty primarily demonstrate dimensional analysis to students but they do not explicitly teach how to perform dimensional analysis (for generating or checking equations) or require students to practice dimensional analysis in course assignments.
\end{abstract}

\section{INTRODUCTION}

A major goal of physics instruction is for students to begin thinking like experts in the field. One expert practice is to consider the dimensions or units of physical quantities when making physical arguments. The dimensions of a quantity indicate its type in terms of fundamental quantities (i.e. length, time, mass, charge). For example, the dimensions of force are $[$ mass $][$ length $] /[\text { time }]^{2}$. The units of a quantity indicate an amount and may vary for a single type of quantity depending on the system of units being used. For example, the SI units of force are Newton's while the cgs units of force are dynes. The purpose of this study is to identify faculty ideas about dimensional analysis and how they teach dimensional analysis to graduate and undergraduate students.

Although nearly all textbooks discuss units and dimensions, very few studies have examined the role of dimensional analysis in physics instruction. Some evidence indicates that explicit instruction in dimensional analysis increases students' problem-solving performance. Warren [1] did a comparison/control study of the effectiveness of evaluation tasks - including dimensional analysis and limiting case analysis - in algebra-based physics and found that students who had used these tasks scored higher on multiple-choice assessments. Similarly, Reed [2] did a comparison/control study in a psychology course and saw improvements in students' abilities to construct equations when given basic instruction on units. No studies have examined instructor views and practices around dimensional analysis.

To better understand the instructional landscape of teaching dimensional analysis, we examine physics experts' views and uses of dimensions and units. Understanding the views and instructional goals of faculty is critical in understanding the context in which students may learn to perform dimensional analysis. We report how physics faculty understand what dimensional analysis is, their characterizations of how they teach students to reason with dimensions, and their perceptions of student difficulties when reasoning about dimensions.

\section{METHODOLOGY}

We interviewed $n=8$ physics faculty at a large, public, doctoral-granting, research-intensive institution. These faculty include a non-tenure-track instructor $(\mathrm{n}=1)$, tenure-track professors $(\mathrm{n}=2)$, and tenured professors $(n=5)$. We refer to all of these instructors and professors as faculty. As a group, they teach a variety of courses across the graduate and undergraduate curricula (see Table I). Many of these courses are taught using activeengagement strategies: several sections of introductory courses are taught in a studio format [3] and upper division physics courses routinely use research-based and research-validated teaching strategies.

The interviews were semi-structured in nature and lasted about an hour. The interview protocol had two parts: first the faculty discussed their views and use of dimensional analysis and then they solved two physics problems with a think-aloud protocol [4]. We report the participants' views of dimensional analysis and their reports of their instructional practices. The planned interview questions can be found in Table II.

We use a phenomenographic methodology in designing the study and analyzing these data. A phenomenographic study uses qualitative methods to understand the experiences and perceptions that subjects have about a particular object [5]. In this study, we use interviews to understand faculty perceptions about dimensional analysis. The goal of a phenomenography is to characterize the variation of experiences around a phenomenon. We expect faculty might teach dimensional analysis differently or expect different student performance across different instructional contexts (e.g. introductory vs. graduate courses or across different research areas), and therefore it was important to interview a set of faculty with a broad range of instructional and research experiences at the university.

The interviews were video and audio recorded and transcripts of the first part of the interview were generated. These transcripts were then read multiple times over several weeks and relevant episodes were identified. A danger of isolating statements is that the meaning of 
TABLE I: Overview of faculty, their typical teaching assignments, and some beliefs reported here. Faculty who expressed a belief or made a statement similar to/in contradiction to these topics are coded as Yes/No. Faculty who did not address a particular topic are coded as "N/A".

\begin{tabular}{|c|c|c|c|c|c|c|c|c|}
\hline \multirow[t]{2}{*}{ Faculty } & \multicolumn{3}{|c|}{ Level of Courses Taught } & \multicolumn{2}{|c|}{$\begin{array}{l}\text { Faculty self reported def- } \\
\text { inition of dimensional } \\
\text { analysis }\end{array}$} & \multirow[t]{2}{*}{$\begin{array}{l}\text { Thinks about Di- } \\
\text { mensions \& Units } \\
\text { Interchangeably }\end{array}$} & \multirow[t]{2}{*}{$\begin{array}{l}\text { Some Dissat- } \\
\text { isfaction with } \\
\text { Instruction }\end{array}$} & \multirow{2}{*}{$\begin{array}{l}\text { Believes } \\
\text { Students } \\
\text { Have } \\
\text { Difficulties }\end{array}$} \\
\hline & $\begin{array}{l}\text { Intro/ } \\
\text { Modern }\end{array}$ & $\begin{array}{l}\text { Upper } \\
\text { Division }\end{array}$ & Grad & $\begin{array}{l}\text { Generative } \\
\text { Dimensional } \\
\text { Analysis }\end{array}$ & $\begin{array}{l}\text { Checking } \\
\text { Units/ } \\
\text { Dimensions }\end{array}$ & & & \\
\hline Cody & $\mathrm{X}$ & & & Yes & Yes & Yes & $\mathrm{N} / \mathrm{A}$ & Yes \\
\hline Perry & $\mathrm{X}$ & & & Yes & Yes & Yes & Yes & Yes \\
\hline Devin & $\mathrm{X}$ & $\mathrm{X}$ & $\mathrm{X}$ & Yes & Yes & No & $\mathrm{N} / \mathrm{A}$ & No \\
\hline Harley & & $\mathrm{X}$ & & Yes & $\begin{array}{l}\text { Discussed } \\
\text { with } \\
\text { prompting }\end{array}$ & $\mathrm{N} / \mathrm{A}$ & $\mathrm{N} / \mathrm{A}$ & Yes \\
\hline Erin & & $\mathrm{X}$ & $\mathrm{X}$ & Yes & Yes & No & Yes & $\mathrm{N} / \mathrm{A}$ \\
\hline Reese & & $\mathrm{x}$ & $\mathrm{X}$ & Yes & Yes & Yes & Yes & No \\
\hline Drew & & $\mathrm{X}$ & $\mathrm{X}$ & Yes & $\begin{array}{l}\text { Discussed } \\
\text { with } \\
\text { prompting }\end{array}$ & Yes & Yes & No \\
\hline Joni & & $\mathrm{X}$ & $\mathrm{X}$ & Yes & $\mathrm{N} / \mathrm{A}$ & $\mathrm{N} / \mathrm{A}$ & $\mathrm{N} / \mathrm{A}$ & $\mathrm{N} / \mathrm{A}$ \\
\hline
\end{tabular}

TABLE II: Planned interview questions. This paper includes analysis of questions 1,4 , and 6. Question 6 was only asked of 6 of the 8 faculty.

\begin{tabular}{ll}
\hline \hline$\#$ & Question \\
\hline $1^{*}$ & What does "doing dimensional analysis" mean to \\
& you? (unit analysis, can you expand on that?) \\
2 & $\begin{array}{l}\text { Do you use dimensional analysis when working on } \\
\text { research problems? How? }\end{array}$ \\
3 & $\begin{array}{l}\text { Do you instruct or discuss dimensional analysis with } \\
\text { the students that you supervise? }\end{array}$ \\
$4^{*}$ & $\begin{array}{l}\text { In the classes that you teach, do you discuss dimen- } \\
\text { sional analysis? Demonstrate it? Assign homework }\end{array}$ \\
problems? & $\begin{array}{l}\text { Under what circumstances do you particularly want } \\
\text { students to be thinking about units or dimensions to } \\
\text { make sense out of a situation? }\end{array}$ \\
$6^{*}$ & $\begin{array}{l}\text { Have you noticed student difficulties with using units } \\
\text { or dimensions? }\end{array}$ \\
7 & $\begin{array}{l}\text { Do you expect students to be doing dimensional } \\
\text { analysis for our assignments/exam without explicit } \\
\text { prompting? } \\
\text { What is your favorite order of magni- } \\
\text { tude/estimation/Fermi problem? } \\
\text { your favorite? }\end{array}$
\end{tabular}

the episode might be misinterpreted without a larger context. To mitigate this danger, we generated a paragraph description of each statement when pulling the statement out of the transcript. We discussed the interpretation of each statement included in this report until a consensus interpretation was reached, referring back to the full transcript when necessary. In a phenomenographic study, the researcher must make every attempt to separate their own beliefs about the object under investigation from the beliefs of the subjects. By attending to the context of each statement and by reaching consensus about the meaning of each statements, we attempt to be as faithful as possible to the meanings the faculty tried to convey.

\section{RESULTS}

\section{A. Generative dimensional analysis vs. unit checking}

For five of the faculty (Erin, Perry, Cody, Reese, Devin), the term dimensional analysis refers to two processes. It can either mean (1) deriving an equation by considering the dimensions of quantities that are likely relevant to the system (a generative dimensional analysis), or (2) checking the units or dimensions of a result to make sure it is the expected type of quantity (unit checking). Erin characterized the generative dimensional analysis process to be the more proper definition: "[dimensional analysis] means two different things. There's one thing it means, which is not usually what I mean but it's what I sometimes think of as the proper dimensional analysis, which is attempting to solve a problem, or constrain a problem, by knowing what the dimensions of the 
relevant quantities are. But the common use of it for me is to check that an answer might be correct by checking that the dimensions work out."

Two faculty members (Harley and Drew) initially gave the generative definition but discussed unit checking when prompted specifically with the term "unit checking'. For example, Harley said, "If you call it unit analysis I would think more of, I derive these equations or equation and I don't know if it's correct, let's at least check the units."

One faculty member (Joni) did not talk about unit checking and was not prompted to discuss it. All seven of the other faculty expressed the importance of checking for errors using units or dimensions. For example, Erin said, "It's something I remember my freshman professor pointing out that, he's like, 'you get hardly anything off if you just make a little mistake somewhere in your math but if your units don't match up, or your dimensions, then you get a lot off. It means you have no idea what you're doing." Throughout the rest of this manuscript the unit/dimension checking definition is discussed in the context of both checking a final value and checking algebra in the middle of a problem.

Four of the faculty (Reese, Cody, Perry, Drew) talk about dimensions and units interchangeably. Reese explained "Actually only recently I became aware that I was mixing up units and dimensions...They [dimensions and units] were interchangeable in my mind for a long time. I often find it easier to work in units even though I call it dimensional analysis."

Harley is the only faculty member we interviewed who stated a dislike of generative dimensional analysis. Harley views it as a cheat and prefers rigorous derivation. "I actually try to avoid using dimensional analysis. If I can give a complete demonstration of something, I'm much happier... I'm not a big fan of [generative dimensional analysis]." However, Harley values unit checking. Harley stated that "In homework or on an exam [students] don't do the check and I see because they don't have the right units. I think it's not a horrible mistake but it's a pretty serious one because I think a physicist always has to think about the units."

\section{B. Instruction mainly focuses on unit checking through demonstration}

Although faculty expect students to do dimensional analysis (particularly in the sense of unit checking), the faculty generally described their instruction of dimensional analysis as demonstration, but they do not require students to perform dimensional analysis. For example, Perry said, in lecture, "I often will try to keep the units in and then I will stare at it and say 'oh my gosh I dropped a factor of something here, where did I drop it?' and then I can figure it out . So I demonstrate why [unit checking is] useful in real time." Reese demonstrates both generative dimensional analysis and unit checking in class. When discussing the solutions to short quizzes in a graduate course, Reese said "I'll give the class a quiz so that they're doing something themselves for about five minutes and then [I'll] go over the answer on the board. And... I'll try to point out when there are ways of checking the answer by checking the dimensions. Or if there's a formula we needed to remember to get the answer, how I can use dimensional reasoning to say this must be the formula, or at least it has this form plus or minus a numerical constant. So yeah, I try to point out how I'm thinking about it so that they have some exposure to it." However, Reese's instruction is limited to demonstration in class: "I haven't written problems that instruct them on how to do it properly or do it at all." Erin's instruction of dimensional analysis happens most often as demonstration to individuals on homework or exams. "It often, I think, comes up when I'm looking at students' answers and I use dimensional analysis to check their answers but that's different from teaching the entire class."

Interestingly, Joni encourages students to use generative dimensional analysis but did not discuss instruction on unit checking during the interview. Joni reported showing one or two examples of generative dimensional analysis in class typically but considers generative dimensional analysis to be mainly a tool for students to use on homework and exams. Joni reported writing problems that are easier to solve using generative dimensional analysis and encouraging (but not requiring) students to use a generative dimensional analysis. Students "don't have to use [dimensional analysis] but if they use it, they get a lot of, it's much quicker."

Half of the interviewees (Erin, Perry, Reese, and Drew) believe they do not adequately highlight this tool in their instruction. For example, Reese said, "There may be a contradiction in what I teach and what I expect." Erin said, "Yes, I do expect students to do dimensional analysis and I think it is something that I should probably be more conscious about." Perry said, "I probably should have done more." Drew said, "I'm pretty sure students have a lot of difficulties. And [dimensional analysis] is kind of really tricky. I mean, I haven't thought about the point of where we should teach [dimensional analysis], at what level."

\section{Faculty beliefs about student difficulties}

Faculty views about whether students had difficulties using units were not uniform. Three faculty (Cody, Harley and Perry) said that students did not have difficulties with units. For example, Harley stated, "I haven't seen [difficulties with units] as a pattern... no repetitive pattern that really worries [me]." Cody believes students are very comfortable with units, saying, "I think units, for students, tend to be a kind of source of security...and when they don't necessarily know what else they're doing, when they're crunching numbers or working through the algebra... a lot of them will start the term by drag- 
ging the units right through their algebra."

Although we ultimately code Perry as believing that students have few/no difficulties with units, Perry's response was difficult to categorize. At first, Perry stated that students were doing "much better than expected" with units. When pressed further, Perry said that, in a modern physics class, "I had rather clever students coming in [to office hours] and being very confused about what the heck was going on with the units." At the beginning of the course, problems gave energies in units of Joules but later, when doing quantum mechanics problems, problems gave energies in units of MeV. Perry said that after the second homework, students begin to pick up on this switch of units and that this contributed to Perry's overall view of the students doing well.

The other three faculty (Reese, Devin and Drew) believe students have difficulty with dimensional analysis. For example Reese stated, "I'm pretty sure there is evidence of it [difficulties] but I haven't been paying attention." However, these three faculty did not give specific examples of student difficulties.

Devin, Erin, and Harley talked about noticing that students do not check the units of their solutions. For example, Devin spoke about how introductory physics students commonly note on their answers to homework problems "the dimensions are correct, even if they're not... half of them just write it down." Devin's statement is in reference to prompted reflection in written homework assignments. Erin and Harley spoke of their observations while grading homework and exams. These two faculty could spot errors in calculations using unit checking that the students could have spotted using the same tool. In Erin and Harley's descriptions they reference homework assignments where reflections are not explicitly required.

\section{LIMITATIONS}

This study reports the views of a small number of physics faculty from a single pubic, research-intensive institution. Although the academic backgrounds of faculty at different types of institutions (e.g. community colleges, liberal arts colleges, private universities, etc) are similar, the reader is cautioned to not generalize the results of this study to faculty at different types of institutions with different instructional cultures. These data about instructional practices is self-reported. We made no formal, direct observations of instruction as part of this study, but these data are consistent with our knowl- edge of teaching in the department. These data about student difficulties are faculty perceptions of student difficulties, and we make no claims as to how well these perceptions are aligned with students' reasoning or performance.

\section{IMPLICATIONS FOR INSTRUCTION AND CONCLUSIONS}

The term "dimensional analysis" is ambiguous to the experts in this study. We suggest using the language "generative dimensional analysis" and "unit/dimension checking." Faculty should use care to disambiguate "units" and "dimensions" in their language, particularly when talking to more advanced students.

The faculty in this study primarily teach dimensional analysis through demonstration and without requiring students to follow-up with practice. Several of the faculty acknowledged that this approach is probably less effective than is appropriate for this important problem solving tool. Although there is some evidence in the research literature that explicit instruction in dimensional analysis is beneficial to students, there is little research about the effectiveness of a demonstration-only approach. We encourage faculty to critically examine how they instruct students in dimensional analysis and to align their instructional practices with their expectations and goals for student performance.

The faculty in this study generally described dimensional analysis as two processes: a way of generating or constraining equations and as a tool for checking for errors. The faculty self-reported that they generally demonstrate dimensional analysis techniques and expect or encourage students to use them at all levels of university instruction but rarely require it on either homework or exams. The interviews indicated that the faculty generally do not notice or attend to student difficulties with units or dimensions. Some faculty reported that students have difficulties using dimensions and units but no specific student difficulties were identified other than students not checking units at all. Other faculty reported that students generally do not have difficulties using units.

Follow-up studies should include physics faculty at many different types of institutions or faculty is other STEM disciplines. Other studies should include direct observations of classroom practices around dimensional analysis. We intend to investigate student reasoning about dimensions and units, as well as other evaluation strategies, in both introductory and advanced undergraduate physics courses.
[1] A. R. Warren, Impact of Teaching Students to Use Evaluation Strategies, Phys. Rev. ST Phys. Educ. Res. 6 (2010).

[2] S. K. Reed, Does Unit Analysis Help Students Construct Equations?, Cognition Instruct. 24, 3 (2006).

[3] T. Hogue, "New OSU physics studio uses technology to engage student-driven learning." Retrieved June, 2016

[4] K. Ericsson and H. Simon, Verbal reports as data, Psychol. Rev. 87, 3 (1980).

[5] F. Marton, Phenomenography - describing conceptions of the world around us, Instr. Sci. 10, 2 (1981). 